\title{
STUDY OF THE VACUUM MATRIX ELEMENT OF PRODUCTS OF PARAFIELDS
}

\author{
O.W. Greenberg $]^{1}$ \\ Center for Fundamental Physics \\ Department of Physics \\ University of Maryland \\ College Park, MD 20742-4111 \\ and \\ A.K. Mishra 2 \\ Institute of Mathematical Sciences \\ Chennai 600 113, India \\ University of Maryland Preprint PP-09-049 \\ arXiv:0909.4069
}

\begin{abstract}
We study the vacuum matrix elements of products of parafields using graphical and combinatorial methods.
\end{abstract}

\section{Introduction}

We can calculate matrix elements of parafields using either the trilinear commutation relations of Green, or using the Green ansatz, also due to Green in the same paper [1]. We recently gave a path integral quantization of parastatistics [2], primarily to show that parastatistics can be quantized using path integrals. We found that, in both the canonical and path integral formalisms, the Green ansatz is convenient for calculating quantities in parafield theories. This is in contrast with the trilinear commutation relations that are cumbersome to use for calculations.

${ }^{1}$ email address: owgreen@umd.edu.

${ }^{2}$ email address: mishra@imsc.res.in 
As far as we know, there is no general calculation of the vacuum matrix element of an arbitrary product of parafields in the literature. Such a calculation will be useful in applications of parastatistics, such as a calculation of the partition function and its connection with the counting of partitions in number theory [3]. In addition this calculation presents combinatorial problems that are interesting in their own right. In this paper we present the explicit calculation of the vacuum matrix element of products of $N \leq 6$ scalar parabose fields (and spinor parafermi fields) of any order $p$. We also give examples of terms in the vacuum matrix elements of $N=8$ and $N=12$ parafields. In addition we give methods that are helpful to calculate the vacuum matrix elements to arbitrary order.

\section{The vacuum matrix element of a product of $N$ parabose fields of order $p$}

The matrix element of an arbitrary product of free bose fields is the sum over all products of contractions, each of which is a two-point function with coefficient one. This is the "factor pairing theorem." [4, 5]

For the corresponding case with parabose fields, not all coefficients are one; indeed the main difficulty in giving a general result for parabose fields is to calculate the coefficient of each term. These coefficients are positive or negative integers or zero.

When we work in $x$-space we use the Pauli-Jordan commutator function which is odd in $x$,

$$
i \Delta(x)=\frac{1}{(2 \pi)^{3}} \int d^{4} k \epsilon\left(k^{0}\right) \delta\left(k^{2}-m^{2}\right) \exp (-i k \cdot x)
$$

and

$$
i \Delta(x)=\Delta^{(+)}(x)-\Delta^{(-)}(x)
$$

where

$$
\Delta^{( \pm)}(x)=\int d^{4} k \theta\left( \pm k^{0}\right) \delta\left(k^{2}-m^{2}\right) \exp (-i k \cdot x)
$$

For completeness we also define the even function of $x$

$$
\Delta^{(1)}(x)=\Delta^{(+)}(x)+\Delta^{(-)}(x)
$$


The analogous formulas for free spinor parafermi fields are

$$
\begin{aligned}
i S(x) & =\left(i \nabla_{x}+m\right) i \Delta(x), \\
i S(x) & =S^{(+)}(x)-S^{(-)}(x)
\end{aligned}
$$

where

$$
\begin{gathered}
S^{( \pm)}(x)=\left(i \not_{x}+m\right) i \Delta^{( \pm)}(x) . \\
S^{(1)}(x)=S^{(+)}(x)+S^{(-)}(x) .
\end{gathered}
$$

The formula that we gave in our previous paper,

$$
\phi^{(\alpha)}\left(x_{1}\right) \phi^{(\beta)}\left(x_{2}\right)=\left(2 \delta_{\alpha \beta}-1\right) \phi^{(\beta)}\left(x_{2}\right) \phi^{(\alpha)}\left(x_{1}\right)+\delta_{\alpha \beta} i \Delta\left(x_{1}-x_{2}\right)
$$

is very useful for this calculation. In the present context,

$$
\left(2 \delta_{\alpha_{i} \alpha_{j}}-1\right)^{2}=4 \delta_{\alpha_{i} \alpha_{j}}-4 \delta_{\alpha_{i} \alpha_{j}}+1=1 .
$$

This is obvious without calculation, since

$$
2 \delta_{\alpha_{i} \alpha_{j}}-1=1, i=j \text {, and }-1, i \neq j ;
$$

i.e., this expression has the value \pm 1 and thus its square is 1 . This simple identity plays an important role. It guarantees that the result is independent of the details of the procedure used to evaluate the matrix element and also is useful in evaluating the sums over the $\alpha$ 's. (Another way to write this expression is $2 \delta_{\alpha_{i} \alpha_{j}}-1=(-1)^{\delta_{\alpha \beta}+1}$.) Here the $\left\{\phi^{(\alpha)}\right\}$ fields are the Green components associated with the parabose fields $\{\Phi\}$. The analogous formula for parafermi fields is

$$
\psi^{(\alpha)}\left(x_{1}\right) \bar{\psi}^{(\beta)}\left(x_{2}\right)=\left(1-2 \delta_{\alpha \beta}\right) \bar{\psi}^{(\beta)}\left(x_{2}\right) \psi^{(\alpha)}\left(x_{1}\right)+\delta_{\alpha \beta} i S\left(x_{1}-x_{2}\right)
$$

We choose the following systematic way to evaluate a vacuum matrix element, which we illustrate for the four-point function 3

$$
\begin{aligned}
& \left\langle 0\left|\Phi\left(x_{1}\right) \Phi\left(x_{2}\right) \Phi\left(x_{3}\right) \Phi\left(x_{4}\right)\right| 0\right\rangle= \\
& \sum_{\alpha^{\prime} s}\left\langle 0\left|\phi^{\left(\alpha_{1}\right)}\left(x_{1}\right) \phi^{\left(\alpha_{2}\right)}\left(x_{2}\right) \phi^{\left(\alpha_{3}\right)}\left(x_{3}\right) \phi^{\left(\alpha_{4}\right)}\left(x_{4}\right)\right| 0\right\rangle
\end{aligned}
$$

\footnotetext{
${ }^{3}$ For completeness we record the result for the two-point functions,

$$
\begin{aligned}
& <0\left|\Phi\left(x_{1}\right) \Phi\left(x_{2}\right)\right| 0>=p \Delta^{(+)}\left(x_{2}-x_{1}\right) \\
& <0\left|\Psi\left(x_{1}\right) \bar{\Psi}\left(x_{2}\right)\right| 0>=p S^{(+)}\left(x_{2}-x_{1}\right)
\end{aligned}
$$
}


Let $\phi^{(\alpha)}\left(\equiv \phi^{(\alpha)(+)}+\phi^{(\alpha)(-)}\right)$ act to the left on the vacuum, so that the positive frequency part, i.e. the creation part $\phi^{(\alpha)(-)}$, annihilates the vacuum on the left. Then in Eq. (1) the $i \Delta$ function will be replaced by the $\Delta^{(+)}$function. Now move $\phi^{\left(\alpha_{1}\right)(+)}$ to the right until it annihilates the vacuum on the right. This field will contract with each of the three fields on its right, giving a $\delta_{\alpha_{1} \alpha_{j}}$ factor multiplying a matrix element with a product of two fields plus a term in which $\phi$ has moved one transposition to the right multiplied by a $(2 \delta-1)$ factor. The remaining twopoint functions can now be evaluated in a similar manner, except that the second type of term just described with the $(2 \delta-1)$ factor will be absent. Where a factor $\left(2 \delta_{\alpha_{i} \alpha_{j}}-1\right)^{2}$ occurs, we replace it by 1 according to Eq. 110$)$. For our illustrative case of the four-point function, this procedure, which we call the first step in our general discussion below, yields

$$
\begin{aligned}
& \left\langle 0\left|\Phi\left(x_{1}\right) \Phi\left(x_{2}\right) \Phi\left(x_{3}\right) \Phi\left(x_{4}\right)\right| 0\right\rangle= \\
& \sum_{\alpha^{\prime} s} \delta_{\alpha_{1} \alpha_{2}} \Delta^{(+)}\left(x_{1}-x_{2}\right) \delta_{\alpha_{3} \alpha_{4}} \Delta^{(+)}\left(x_{3}-x_{4}\right)+ \\
& \quad\left(2 \delta_{\alpha_{1} \alpha_{2}}-1\right) \delta_{\alpha_{1} \alpha_{3}} \Delta^{(+)}\left(x_{1}-x_{3}\right) \delta_{\alpha_{2} \alpha_{4}} \Delta^{(+)}\left(x_{2}-x_{4}\right)+ \\
& \left(2 \delta_{\alpha_{1} \alpha_{2}}-1\right)\left(2 \delta_{\alpha_{1} \alpha_{3}}-1\right) \delta_{\alpha_{1} \alpha_{4}} \Delta^{(+)}\left(x_{1}-x_{4}\right) \delta_{\alpha_{2} \alpha_{3}} \Delta^{(+)}\left(x_{2}-x_{3}\right) .
\end{aligned}
$$

For the second step of the calculation of this four-point function, each term has two different factors $\delta_{\alpha_{i} \alpha_{j}}$ that we use to do two of the sums over the $\alpha$ 's. By renaming the dummy $\alpha$ 's, if necessary, we reduce the result to a sum over the first two $\alpha$ 's. The result is

$$
\begin{aligned}
& \left\langle 0\left|\Phi\left(x_{1}\right) \Phi\left(x_{2}\right) \Phi\left(x_{3}\right) \Phi\left(x_{4}\right)\right| 0\right\rangle= \\
& \quad \sum_{\alpha_{1} \alpha_{2}}\left[\Delta^{(+)}\left(x_{1}-x_{2}\right) \Delta^{(+)}\left(x_{3}-x_{4}\right)+\right. \\
& \quad\left(2 \delta_{\alpha_{1} \alpha_{2}}-1\right) \Delta^{(+)}\left(x_{1}-x_{3}\right) \Delta^{(+)}\left(x_{2}-x_{4}\right)+ \\
& \left.\quad\left(2 \delta_{\alpha_{1} \alpha_{2}}-1\right)\left(2 \delta_{\alpha_{1} \alpha_{2}}-1\right) \Delta^{(+)}\left(x_{1}-x_{4}\right) \Delta^{(+)}\left(x_{2}-x_{3}\right)\right],
\end{aligned}
$$

where we used the identity Eq. 10 in the third term. In the third and final step, 
we do the sums over the two remaining $\alpha$ 's. This gives the result

$$
\begin{aligned}
& \left\langle 0\left|\Phi\left(x_{1}\right) \Phi\left(x_{2}\right) \Phi\left(x_{3}\right) \Phi\left(x_{4}\right)\right| 0\right\rangle= \\
& \quad p^{2} \Delta^{(+)}\left(x_{1}-x_{2}\right) \Delta^{(+)}\left(x_{3}-x_{4}\right)+p(2-p) \Delta^{(+)}\left(x_{1}-x_{3}\right) \Delta^{(+)}\left(x_{2}-x_{4}\right)+ \\
& p^{2} \Delta^{(+)}\left(x_{1}-x_{4}\right) \Delta^{(+)}\left(x_{2}-x_{3}\right) .
\end{aligned}
$$

For charged spinor parafermi fields the calculation is similar, except we keep only contractions between and field $\psi$ and its conjugate $\bar{\psi}$.

In the general case the first step of this procedure reduces the matrix element with $N=2 n$ fields to a sum of $N-1$ terms, each of which will have a product of $N-2$ fields. The entire procedure yields $(N-1)$ !! terms, each of which has the product of $n$ two-point functions $\Delta^{(+)}$multiplied by the product of $n \delta$ 's and a product of $(2 \delta-1)$ factors. These terms will be summed over the $N \alpha$ 's from 1 to $p$ for each $\alpha$.

We represent each term by a graph containing $N$ points and $n$ directed lines or "links" below the points representing the contractions. We can arrive at these graphs directly by forming all possible $(N-1)$ !! distinct combinations of $n$-pairs involving $N=2 n$ integers 1 to $N$. In the graphs, these integers are represented by $N$ points, numbered in ascending order. Subsequently, we connect the pair of points representing the integers in a pair, by a line in the graph. As each combination has $n$ pairs, $n$ such lines are required. We choose the line (or link) to go from the lower numbered point to a higher numbered one.

Next we provide an algebraic correspondence for these graphs. We start with a given graph and associate a factor $\delta_{\alpha_{i} \alpha_{j}} \Delta^{(+)}\left(x_{\alpha_{i}}-x_{\alpha_{j}}\right)$ with the line linking the points $i$ and $j$. We repeat this process for each of the $n$ links, and form the product of all factors $\delta_{\alpha_{k} \alpha_{l}} \Delta^{(+)}\left(x_{\alpha_{k}}-x_{\alpha_{l}}\right)$. This resulting term has to be multiplied by a product of $\{(2 \delta-1)\}$ factors. The number of crossings of the links corresponds to the number of $(2 \delta-1)$ factors in the product. (All of the links must lie below (or above) the line containing the $N$ points.) To arrive at their specific values, start with the line originating from the point 1 , and terminating, say, at point $t$. If this line crosses other lines originating from points $t_{1}, t_{2}, \ldots t_{q}$, all lying to the left of the point $t$, we get a product of $\{(2 \delta-1)\}$ factors, namely, $\left\{\left(2 \delta_{\alpha_{1} \alpha_{j}}-1\right), t_{1} \leq j \leq t_{q}\right\}$. If the line originating from point 1 does not cross any other line, then no $\left(2 \delta_{\alpha_{1} \alpha_{j}}-1\right)$ factor 
is introduced. Repeat this process for the lines originating from points $2,3, \ldots, n$, and for each new crossing encountered in this process, introduce a $(2 \delta-1)$ factor. Finally, take the product of all these $\{(2 \delta-1)\}$ factors, multiply this quantity by the product of $n$-pairs of $\delta_{\alpha_{i} \alpha_{j}} \Delta^{(+)}\left(x_{\alpha_{i}}-x_{\alpha_{j}}\right)$ factors obtained earlier, and sum the resulting expression from 1 to $p$ over $\alpha_{1}, \alpha_{2}, \ldots, \alpha_{N}$.

As an illustration, we consider here the $\mathrm{N}=6$ case. Generating all the five 0-crossing, six 1-crossing, three 2-crossings and the single 3-crossings graphs for $N$ $=6$, and substituting the associated coefficients derived in the earlier paragraph, the six-point function is

$$
\begin{aligned}
\langle 0| \Phi( & \left.x_{1}\right) \Phi\left(x_{2}\right) \Phi\left(x_{3}\right) \Phi\left(x_{4}\right) \Phi\left(x_{5}\right) \Phi\left(x_{6}\right)|0\rangle \\
= & p^{3}\left\{\Delta^{(+)}\left(x_{1}-x_{2}\right) \Delta^{(+)}\left(x_{3}-x_{4}\right) \Delta^{(+)}\left(x_{5}-x_{6}\right)+\Delta^{(+)}\left(x_{1}-x_{2}\right) \Delta^{(+)}\left(x_{3}-x_{6}\right) \Delta^{(+)}\left(x_{4}-x_{5}\right)\right. \\
& +\Delta^{(+)}\left(x_{1}-x_{4}\right) \Delta^{(+)}\left(x_{2}-x_{3}\right) \Delta^{(+)}\left(x_{5}-x_{6}\right)+\Delta^{(+)}\left(x_{1}-x_{6}\right) \Delta^{(+)}\left(x_{2}-x_{3}\right) \Delta^{(+)}\left(x_{4}-x_{5}\right) \\
& \left.+\Delta^{(+)}\left(x_{1}-x_{6}\right) \Delta^{(+)}\left(x_{2}-x_{5}\right) \Delta^{(+)}\left(x_{3}-x_{4}\right)\right\}+ \\
& p^{2}(2-p) \\
\times \quad & \left\{\Delta^{(+)}\left(x_{1}-x_{6}\right) \Delta^{(+)}\left(x_{2}-x_{4}\right) \Delta^{(+)}\left(x_{3}-x_{5}\right)+\Delta^{(+)}\left(x_{1}-x_{5}\right) \Delta^{(+)}\left(x_{2}-x_{3}\right) \Delta^{(+)}\left(x_{4}-x_{6}\right)\right. \\
& +\Delta^{(+)}\left(x_{1}-x_{3}\right) \Delta^{(+)}\left(x_{2}-x_{6}\right) \Delta^{(+)}\left(x_{4}-x_{5}\right)+\Delta^{(+)}\left(x_{1}-x_{3}\right) \Delta^{(+)}\left(x_{2}-x_{4}\right) \Delta^{(+)}\left(x_{5}-x_{6}\right) \\
& \left.+\Delta^{(+)}\left(x_{1}-x_{2}\right) \Delta^{(+)}\left(x_{3}-x_{5}\right) \Delta^{(+)}\left(x_{4}-x_{6}\right)+\Delta^{(+)}\left(x_{1}-x_{5}\right) \Delta^{(+)}\left(x_{2}-x_{6}\right) \Delta^{(+)}\left(x_{3}-x_{4}\right)\right\} \\
& +p(2-p)^{2} \\
\times \quad & \Delta^{(+)}\left(x_{1}-x_{5}\right) \Delta^{(+)}\left(x_{2}-x_{4}\right) \Delta^{(+)}\left(x_{3}-x_{6}\right)+\Delta^{(+)}\left(x_{1}-x_{4}\right) \Delta^{(+)}\left(x_{2}-x_{6}\right) \Delta^{(+)}\left(x_{3}-x_{5}\right) \\
& \left.+\Delta^{(+)}\left(x_{1}-x_{3}\right) \Delta^{(+)}\left(x_{2}-x_{5}\right) \Delta^{(+)}\left(x_{4}-x_{6}\right)\right\} \\
& -p\left(p^{2}-6 p+4\right)\left\{\Delta^{(+)}\left(x_{1}-x_{4}\right) \Delta^{(+)}\left(x_{2}-x_{5}\right) \Delta^{(+)}\left(x_{3}-x_{6}\right)\right\}
\end{aligned}
$$

Next, we give the following 4 cases corresponding to all possible 5-crossings graphs for an 8-point function as illustrations. The algebraic expressions for graph (1), graph (2), graph (3) and graph (4), drawn in figures (1-4), are

$$
\begin{aligned}
& \sum_{\alpha_{1}, \alpha_{2}, \ldots, \alpha_{8}}\left(2 \delta_{\alpha_{1}, \alpha_{2}}-1\right)\left(2 \delta_{\alpha_{1} \alpha_{3}}-1\right)\left(2 \delta_{\alpha_{1} \alpha_{4}}-1\right)\left(2 \delta_{\alpha_{2} \alpha_{3}}-1\right)\left(2 \delta_{\alpha_{2} \alpha_{4}}-1\right) \times \\
& \delta_{\alpha_{1} \alpha_{5}} \Delta^{(+)}\left(x_{1}-x_{5}\right) \delta_{\alpha_{2} \alpha_{6}} \Delta^{(+)}\left(x_{2}-x_{6}\right) \times \\
& \delta_{\alpha_{3}, \alpha_{8}} \Delta^{(+)}\left(x_{3}-x_{8}\right) \delta_{\alpha_{4} \alpha_{7}} \Delta^{(+)}\left(x_{4}-x_{7}\right),
\end{aligned}
$$




$$
\begin{aligned}
& \sum_{\alpha_{1}, \alpha_{2}, \ldots, \alpha_{8}}\left[\left(2 \delta_{\alpha_{1} \alpha_{2}}-1\right)\left(2 \delta_{\alpha_{1} \alpha_{3}}-1\right)\left(2 \delta_{\alpha_{2}, \alpha_{3}}-1\right)\left(2 \delta_{\alpha_{2} \alpha_{5}}-1\right)\left(2 \delta_{\alpha_{3} \alpha_{5}}-1\right) \times\right. \\
& \delta_{\alpha_{1} \alpha_{4}} \Delta^{(+)}\left(x_{1}-x_{4}\right) \delta_{\alpha_{2} \alpha_{6}} \Delta^{(+)}\left(x_{2}-x_{6}\right) \times \\
& \left.\delta_{\alpha_{3} \alpha_{7}} \Delta^{(+)}\left(x_{3}-x_{7}\right) \delta_{\alpha_{5} \alpha_{8}} \Delta^{(+)}\left(x_{5}-x_{8}\right)\right], \\
& \sum_{\alpha_{1}, \alpha_{2}, \ldots, \alpha_{8}}\left[\left(2 \delta_{\alpha_{1} \alpha_{2}}-1\right)\left(2 \delta_{\alpha_{1} \alpha_{3}}-1\right)\left(2 \delta_{\alpha_{1} \alpha_{4}}-1\right)\left(2 \delta_{\alpha_{2} \alpha_{4}}-1\right)\left(2 \delta_{\alpha_{3} \alpha_{4}}-1\right) \times\right. \\
& \delta_{\alpha_{1} \alpha_{5}} \Delta^{(+)}\left(x_{1}-x_{5}\right) \delta_{\alpha_{2} \alpha_{7}} \Delta^{(+)}\left(x_{2}-x_{7}\right) \times \\
& \left.\delta_{\alpha_{3} \alpha_{6}} \Delta^{(+)}\left(x_{3}-x_{6}\right) \delta_{\alpha_{4} \alpha_{8}} \Delta^{(+)}\left(x_{4}-x_{8}\right)\right],
\end{aligned}
$$

and

$$
\begin{aligned}
& \sum_{\alpha_{1}, \alpha_{2}, \ldots, \alpha_{8}}\left[\left(2 \delta_{\alpha_{1} \alpha_{3}}-1\right)\left(2 \delta_{\alpha_{1} \alpha_{4}}-1\right)\left(2 \delta_{\alpha_{2} \alpha_{3}}-1\right)\left(2 \delta_{\alpha_{2} \alpha_{4}}-1\right)\left(2 \delta_{\alpha_{3} \alpha_{4}}-1\right) \times\right. \\
& \delta_{\alpha_{1} \alpha_{6}} \Delta^{(+)}\left(x_{1}-x_{6}\right) \delta_{\alpha_{2} \alpha_{5}} \Delta^{(+)}\left(x_{2}-x_{5}\right) \times \\
& \left.\delta_{\alpha_{3} \alpha_{7}} \Delta^{(+)}\left(x_{3}-x_{7}\right) \delta_{\alpha_{4} \alpha_{8}} \Delta^{(+)}\left(x_{4}-x_{8}\right)\right],
\end{aligned}
$$

respectively.

Since there are $n \delta$-functions in each one of the $(N-1)$ !! terms, in the second step we reduce the expressions to sums over $n \alpha$ 's. We now define a graph with $n$ vertices and as many links between vertices as there are crossings in the diagrams with $N$ points discussed above. A link connects a pair of vertices if lines starting on each of the pair intersect. The most "saturated" graph with the maximum number, $n(n-1) / 2$ links corresponds to a simplex in $n$-dimensional space. This also implies that the coefficients contain at most a product of $[n(n-1) / 2](2 \delta-1)$ factors. In the final step we do the sums over the remaining $n \alpha$ 's. The total number of summations to be carried out is $(N-1)$ !!. Obviously for a graph with $m$ crossings $(1 \leq m \leq n(n-1) / 2)$, the summand is a product of $m(2 \delta-1)$ terms. When $m$ $=0$, summand is unity, and the coefficient equals $p^{n}$. For $m=1$, the summand is $\left(2 \delta_{\alpha_{i}, \alpha_{j}}-1\right)$, and the associated coefficient takes the value $p^{n-1}(2-p)$. When $m=$ 2 , all possible values of the summand, through relabeling the dummy indices $\left\{\alpha_{i}\right\}$, 
get reduced to two forms, viz, $\left(2 \delta_{\alpha_{1}, \alpha_{2}}-1\right)\left(2 \delta_{\alpha_{1}, \alpha_{3}}-1\right)$, and $\left(2 \delta_{\alpha_{1}, \alpha_{2}}-1\right)\left(2 \delta_{\alpha_{3}, \alpha_{4}}-1\right)$. The coefficients corresponding to both the summands are $p^{n-2}(2-p)^{2}$. For $m$ $=3, N=6$, only one graph with three crossings exists; having the summand as $\left(2 \delta_{\alpha_{1}, \alpha_{2}}-1\right)\left(2 \delta_{\alpha_{1}, \alpha_{3}}-1\right)\left(2 \delta_{\alpha_{2}, \alpha_{3}}-1\right)$, and the coefficient is $-p\left(p^{2}-6 p+4\right)$. In general, when $m$ takes the maximum value $n(n-1) / 2$, the is $\prod_{i<j ; 1 \leq i \leq n-1 ; 2 \leq j \leq n}\left(2 \delta_{\alpha_{i}, \alpha_{j}}-1\right)$, and there is only one graph corresponding to this most saturated case.

As a step towards finding a general method to evaluate the coefficients, we can simplify the problem of $(N-1)$ !! summations for $N$ number of fields by enumerating the number of graphs $T_{N m}$ having $m$ numbers of crossings. As noted earlier, $0 \leq$ $m \leq n(n-1) / 2$. In the following table, these values are listed for $2 \leq N \leq 10$. The * in a box implies that the $m$ has reached its maximum permissible value. 


\section{TABLE I}

\begin{tabular}{|c|c|c|c|c|c|c|c|c|c|c|c|c|}
\hline$m$ & 0 & 1 & 2 & 3 & 4 & 5 & 6 & 7 & 8 & 9 & 10 & $\begin{array}{c}\sum_{m} T_{N m} \\
=(N-1) ! !\end{array}$ \\
\hline$T_{2 m}$ & $1^{*}$ & & & & & & & & & & 1 \\
\hline$T_{4 m}$ & 2 & $1^{*}$ & & & & & & & & & & 3 \\
\hline$T_{6 m}$ & 5 & 6 & 3 & $1^{*}$ & & & & & & & & 15 \\
\hline$T_{8 m}$ & 14 & 28 & 28 & 20 & 10 & 4 & $1^{*}$ & & & & & 105 \\
\hline$T_{10 m}$ & 42 & 120 & 180 & 195 & 165 & 117 & 70 & 35 & 15 & 5 & $1^{*}$ & 945 \\
\hline
\end{tabular}

Now we provide a general method for carrying out summation over $\alpha$ 's. We express the sum from 1 to $p$ over each of the $n \alpha$ 's as a sum over the partitions of $n$. In our example of the four-point function, we have reduced the calculation of, for example, the numerical coefficient of the middle term in Eq. (6), to the sum

$$
\sum_{\alpha_{1}, \alpha_{2}=1}^{p}\left(2 \delta_{\alpha_{1}, \alpha_{2}}-1\right)
$$

Instead of summing each $\alpha$ from 1 to $p$, we can break up the sums into a sum over single values of each $\alpha$, for example

$$
\sum_{\alpha_{i}=1}^{p}=\sum_{\alpha_{i}=1}+\sum_{\alpha_{i}=2}+\cdots+\sum_{\alpha_{i}=p}
$$

When we carry out multiple sums, we have to take account whether there is a $(2 \delta-1)$ factor connecting a pair of $\alpha$ 's and which sets of $\alpha$ 's match and which don't match. If there is no $(2 \delta-1)$ factor connecting a pair of $\alpha$ 's there is a factor 1 ; if there is a $(2 \delta-1)$ factor connecting a pair of $\alpha$ 's there is a factor -1 . Next, we select any 
specific term, and sum over the set of, say, $h \alpha$ 's, that do not appear in the summand. Obviously summation over these $h \alpha$ 's leads to a multiplicative factor of $p^{h}$ in the coefficients. Now we are left with the summation over the remaining $(n-h) \equiv f$ $\alpha$ 's. To make further progress, we begin with the case of $h=0$ corresponding to the maximum number of crossings. Here not only all $\alpha$ 's appear in the summand, but each one appears for the equal number of times. This symmetric distribution of $\alpha$ 's in each summand leads to considerable simplification. Our further discussion applies only to this "maximally saturated" case, although the use of partitions with restrictions may apply generally.

For this symmetric case we group the numbers from 1 to $n$ into groups that are equal and unequal. This leads to unrestricted partitions of the number $n$. Let the $p(n)$ partitions of $n$ be labeled $\lambda^{(1)}, \lambda^{(2)}, \cdots, \lambda^{(p(n))}$. For a general $\lambda^{(s)}$ let

$$
\lambda^{(s)}=\left(\lambda_{1}^{(s)} \ldots \lambda_{1}^{(s)} \lambda_{2}^{(s)} \ldots \lambda_{2}^{(s)} \ldots \lambda_{i}^{(s)} \ldots \lambda_{i}^{(s)} \ldots \lambda_{l}^{(s)} \ldots \lambda_{l}^{(s)}\right)
$$

where $m_{i}^{(s)}$ is the number of times the part $\lambda_{i}^{(s)}$ of the partition $\lambda^{(s)}$ occurs, $k^{(s)}=$ $\sum_{i=1}^{l^{(s)}} m_{i}^{(s)}$ is the number of parts of the partition $\lambda^{(s)}, l^{(s)}$ is the number of distinct parts, and the weight is $\left|\lambda^{(s)}\right|=\sum_{(i=1)}^{l^{(s)}} m_{i}^{(s)} \lambda_{i}^{(s)}=n$. Then

$$
\sum_{\alpha_{1}=1}^{p} \cdots \sum_{\alpha_{n}=1}^{p} f\left(\alpha_{1}, \cdots, \alpha_{n}\right)=\left.\sum_{s=1}^{p(n)} \frac{n !}{\prod_{i=1}^{l(s)}\left[\left(\lambda_{i}^{(s)} !\right)^{m_{i}^{(s)}} m_{i}^{(s)} !\right]} \frac{p !}{\left(p-k^{(s)}\right) !} f\left(\alpha_{1}, \cdots, \alpha_{n}\right)\right|_{\lambda_{i}^{(s)}}
$$

where $p^{n}$ is the total number of 'n-tuples' $\left(\alpha_{1}, \alpha_{2}, \ldots, \alpha_{n}\right)$ when each $\alpha$ varies from 1 to $\mathrm{p}$. In our case, the $\left.f\left(\alpha_{1}, \cdots, \alpha_{n}\right)\right|_{\lambda^{(s)}}$ is the product of the factors $(2 \delta-1)$ associated with the crossings discussed above. This product is either 1 or -1 for a given partition. For the case of no crossings, whose $n$-vertex graphs consist of $n$ disconnected points, $\left.f\left(\alpha_{1}, \cdots, \alpha_{n}\right)\right|_{\lambda^{(s)}}=1$ and we find

$$
p^{n}=\sum_{s=1}^{p(n)} \frac{n !}{\prod_{i=1}^{l(s)}\left[\left(\lambda_{i}^{(s)} !\right)^{m_{i}^{(s)}} m_{i}^{(s)} !\right]} \frac{p !}{\left(p-k^{(s)}\right) !}
$$


as we expect. The summand for any 'n-tuple' of $\left(\alpha_{1}, \alpha_{2}, . ., \alpha_{n}\right)$ always reduces to either 1 or -1 . The partitions introduced above determine this sign. As noted above $\left(2 \delta_{\alpha_{i}, \alpha_{j}}-1\right)=1$ if $\alpha_{i}=\alpha_{j}$ and -1 otherwise. If in any given partition $\lambda^{(s)}$, $\alpha_{i}$ is not equal to $\alpha_{j}$ for $A^{(s)}$ number of times, the summand would be $(-1)^{A^{(s)}}$. This is true for all the possible groupings of $\alpha$ 's according to the partition $\lambda_{i}^{(s)}$. This is a consequence of the fact that in the most saturated case the $\alpha$ 's have a symmetric distribution in the summand. From Eq. (25), we get the total number of configurations $E^{(s)}$ associated with the $\lambda^{(s)}$ as

$$
E^{(s)}=X_{n}^{(s)} \frac{p !}{\left(p-k^{(s)}\right) !} ; X_{n}^{(s)}=\frac{n !}{\prod_{i=1}^{l(s)}\left[\left(\lambda_{i}^{(s)} !\right)^{m_{i}^{(s)}} m_{i}^{(s)} !\right]}
$$

The summand contains a product of $n(n-1) / 2\left(2 \delta \alpha_{i}, \alpha_{j}-1\right)$ terms, and in the partition $\lambda^{(s)}$, the number of times for which $\alpha_{i}$ equals $\alpha_{j}$ is

$$
\bar{A}^{(s)}=\sum_{i=1}^{l} m_{i}^{(s)} \frac{\lambda_{i}^{(s)}\left(\lambda_{i}^{(s)}-1\right)}{2} ; \lambda_{i}^{(s)} \geq 2 .
$$

Consequently,

$$
A^{(s)}=\frac{n(n-1)}{2}-\bar{A}^{(s)}
$$

and the coefficient

$$
\sum_{\alpha_{1}, \ldots, \alpha_{n}} \prod_{i<j ; 2 \leq\{i+1, j\} \leq n}\left(2 \delta_{\alpha_{i}, \alpha_{j}}-1\right)=\sum_{s=1}^{p(n)}(-1)^{A^{(s)}} E^{(s)}
$$

As an illustration, we evaluate the coefficient $C_{15}^{12}$ for the most saturated graph corresponding to $N=12$, viz.,

$$
C_{15}^{12}=\sum_{\alpha_{1}, \ldots, \alpha_{6}} \prod_{i<j ; 2 \leq\{i+1, j\} \leq 6}\left(2 \delta_{\alpha_{i}, \alpha_{j}}-1\right)
$$

It contains a product of $15(2 \delta-1)$ factors or a sum of $2^{15}$ terms. The relevant parameters for calculating $C_{15}^{12}$ are given in the TABLE II. Using Eq. (32) and 
Table II, we get

$$
C_{15}^{12}=-p(p-2)^{2}\left(p^{3}-26 p^{2}+152 p-128\right) .
$$

For bosons, $p=1$, and $C_{15}^{12}$ should, and does, reduce to 1 . 
TABLE II

\begin{tabular}{|c|c|c|c|c|c|c|c|}
\hline$s$ & $\lambda^{(s)}$ & $\lambda_{1}^{(s)} / m_{1}^{(s)}$ & $\lambda_{2}^{(s)} / m_{2}^{(s)}$ & $\lambda_{3}^{(s)} / m_{3}^{(s)}$ & $k^{(s)}$ & $E^{(s)}$ & $A^{(s)}$ \\
\hline 1 & {$[6]$} & $6 / 1$ & - & - & 1 & $6 ! p ! /\{6 !(p-1) !\}$ & 0 \\
\hline 2 & {$[5,1]$} & $5 / 1$ & $1 / 1$ & - & 2 & $6 ! \mathrm{p} ! /\{5 !(\mathrm{p}-2) !\}$ & 5 \\
\hline 3 & {$[4,2]$} & $4 / 1$ & $2 / 1$ & - & 2 & $6 ! \mathrm{p} ! /\{4 ! 2 !(\mathrm{p}-2) !\}$ & 8 \\
\hline 4 & {$[4,1,1]$} & $4 / 1$ & $1 / 2$ & - & 3 & $6 ! \mathrm{p} ! /\{4 ! 2 !(\mathrm{p}-3) !\}$ & 9 \\
\hline 5 & {$[3,3]$} & $3 / 2$ & - & - & 2 & $6 ! \mathrm{p} ! /\left\{3 !^{2} 2 !(\mathrm{p}-2) !\right\}$ & 9 \\
\hline 6 & {$[3,2,1]$} & $3 / 1$ & $2 / 1$ & $1 / 1$ & 3 & $6 ! \mathrm{p} ! /\{3 ! 2 !(\mathrm{p}-3) !\}$ & 11 \\
\hline 7 & {$[3,1,1,1]$} & $3 / 1$ & $1 / 3$ & - & 4 & $6 ! \mathrm{p} ! /\left\{3 !^{2}(\mathrm{p}-4) !\right\}$ & 12 \\
\hline 8 & {$[2,2,2]$} & $2 / 3$ & - & - & 3 & $6 ! \mathrm{p} ! /\left\{2 !^{3} 3 !(\mathrm{p}-3) !\right\}$ & 12 \\
\hline 9 & {$[2,2,1,1]$} & $2 / 2$ & $1 / 2$ & - & 4 & $6 ! \mathrm{p} ! /\left\{2 !^{4}(\mathrm{p}-4) !\right\}$ & 13 \\
\hline 10 & {$[2,1,1,1,1]$} & $2 / 1$ & $1 / 4$ & - & 5 & $6 ! \mathrm{p} ! /\{2 ! 4 !(\mathrm{p}-5) !\}$ & 14 \\
\hline 11 & {$[1,1,1,1,1,1]$} & $1 / 6$ & - & - & 6 & $6 ! \mathrm{p} ! /\left\{1 !^{6} 6 !(\mathrm{p}-6) !\right\}$ & 15 \\
\hline
\end{tabular}

\section{Summary and Conclusions}

We used Green's ansatz to evaluate the vacuum matrix element of an arbitrary product of parabose fields of order $p$. We gave a graphical method to reduce an $N=2 n$ point function to a sum over products of $n 2$-point functions. We expressed 
the coefficients of each these $n$ 2-point functions as a sum over $n \alpha_{i}$ 's $\left(1 \leq \alpha_{i} \leq p\right)$. The corresponding graph uniquely determines both the $n 2$-point functions and the coefficients that occur in the sum.

Subsequently, we gave a combinatorial method, based on the partitions of the number $n$, to determine the coefficients. We illustrated this method by the explicit determination of the coefficient for most saturated graph corresponding to the $N=12$ case which involves a sum of $2^{15}$ terms.

\section{Figure Captions}

Figure 1. Case (1) of a 5-crossings graph for an 8-point function. The algebraic expression associated with this graph is given in Eq. (20)

Figure 2. Case (2) of a 5-crossings graph for an 8-point function. The algebraic expression associated with this graph is given in Eq. (21)

Figure 3. Case (3) of a of 5-crossings graph for an 8-point function. The associated algebraic expression for this graph is given in Eq. (22)

Figure 4. Case(4) of a of 5-crossings graph for an 8-point function. The associated algebraic expression for this graph is given in Eq. (23)

\section{Acknowledgements:}

This work was supported in part by the National Science Foundation, Grant No. PHY-0140301 and by the Department of Science and Technology of India Grant. No. DST/INT/US (NSF-RP086).

\section{References}

[1] H.S. Green, Phys. Rev. 90, 270 (1953).

[2] O.W. Greenberg and A.K. Mishra, Phys. Rev. D 70, 125013 (2004). 
[3] M.N. Tran, M.V.N. Murthy, and R.K. Bhaduri, Ann. Phys. 311, 204 (2004).

[4] F.J. Dyson, Phys. Rev. 75, 486, 1736 (1949).

[5] G.C. Wick, Phys. 80, 268 (1950). 


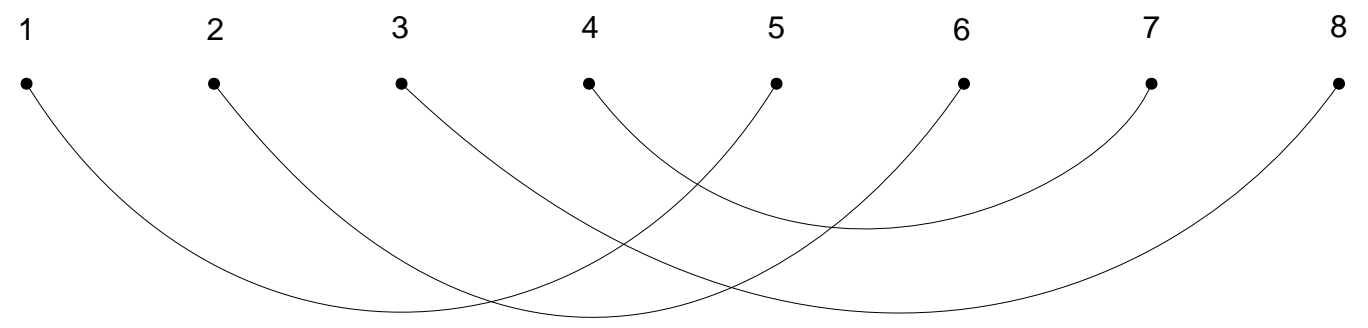

Fig. 1 


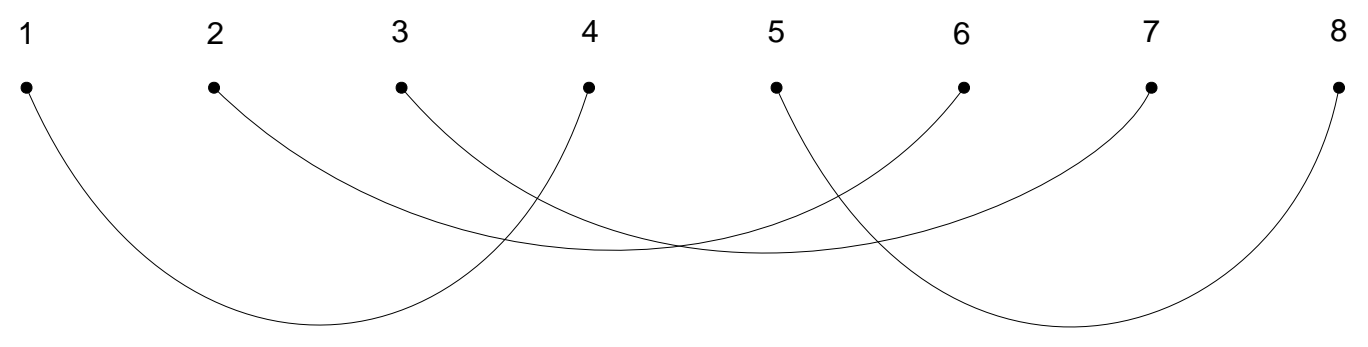

Fig. 2 


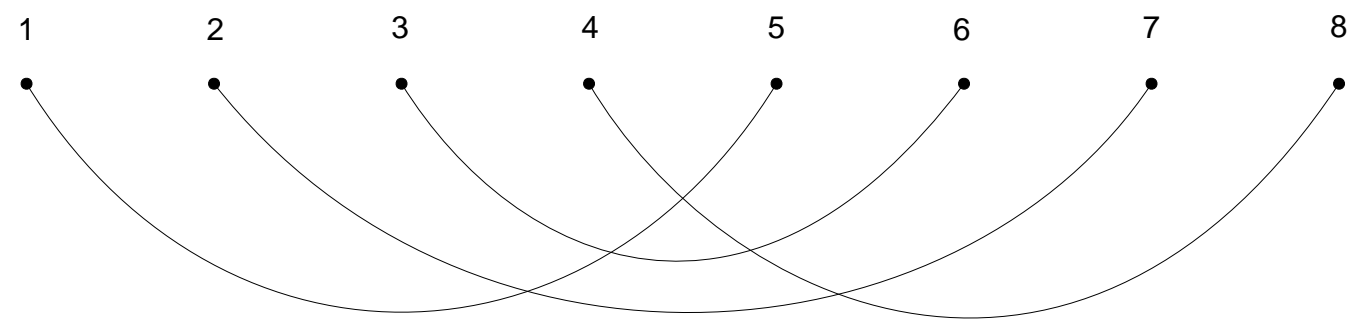

Fig. 3 


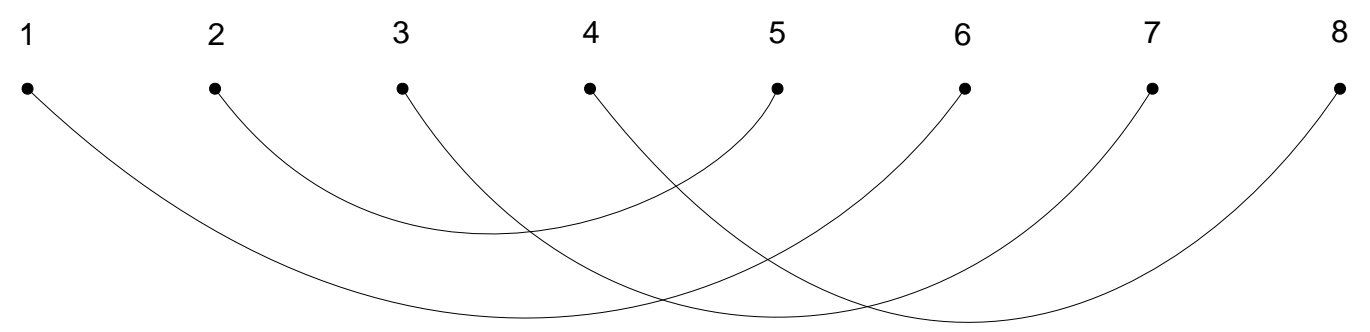

Fig. 4 